\title{
METAFÍSICA Y REALIDAD VIRTUAL. ¿PUEDEN SER REALIDADES VIRTUALES EL UNIVERSO Y LA MENTE? ${ }^{1}$
}

\author{
METAPHysics aND VIRTUAL REALITY. CAN THE UNIVERSE \\ AND THE MIND BE VIRTUAL REALITIES?
}

Manuel Liz Gutiérrez

DOI: $10.26754 /$ ojs_arif/arif.202115314

\begin{abstract}
RESUMEN
Las nuevas tecnologías están cambiando nuestro mundo. Y la filosofía no puede mantenerse al margen. Tampoco sus partes más especulativas. En este trabajo analizamos la noción de realidad virtual en relación con cuatro significados básicos. Lo virtual puede relacionarse con la idea de apariencia, de potencialidad, de mediación y de construcción. En conexión con esa densidad de significado, exploramos varias posibilidades de virtualidad para dos casos límite: el Universo y la mente. Y defendemos tres tesis sobre la realidad virtual: 1) que una realidad virtual total debería ser generada de manera completamente no habitual desde nuestros puntos de vista, 2) que lo virtual se resiste a admitir una distinción apariencia/realidad, y 3) que la posibilidad de que todo el Universo sea una realidad virtual total nos obliga a asumir la verdad de la inteligencia artificial en sentido fuerte.
\end{abstract}

PALABRAS CLAVE: Nuevas tecnologías; metafísica; realidad virtual; simulación; apariencia; potencialidad; mediación; construcción; Universo; mente; inteligencia artificial.

\section{ABSTRACT}

New technologies are changing our world. And philosophy cannot keep out. Neither can its more speculative parts. In this paper, we analyze the notion of virtual reality in relation to four basic meanings. The virtual can be related with the idea of appearance,

\footnotetext{
1 Este trabajo se ha llevado a cabo en el marco del Proyecto de Investigación Personal Perspectives. Concepts and applications (FFI2018-098254-B100), financiado por el Ministerio de Economía y Competitividad del Gobierno de España. Debemos agradecer a Andrés Jaume sus numerosos y siempre oportunos comentarios al mismo.
}

Recibido: 13/04/2021. Aceptado: 24/06/2021

Análisis. Revista de investigación filosófica, vol. 8, n. ${ }^{\circ} 1$ (2021): 47-72

ISSNe: 2386-8066

Copyright: Este es un artículo de acceso abierto distribuido bajo una licencia de uso y distribución "Creative Commons Reconocimiento No-Comercial Sin-Obra-Derivada 4.0 Internacional" (CC BY NC ND 4.0) 
of potentiality, of mediation and of construction. In connection with that density of meaning, we explore several possibilities of virtuality for two limit cases: the Universe and the mind. And we argue for three theses about virtual reality: 1) that a total virtual reality should be generated in a completely unusual way from our points of view, 2) that the virtual refuses to admit an appearance/reality distinction, and 3) that the possibility that the entire Universe is a total virtual reality forces us to assume the truth of artificial intelligence in a strong sense.

KEYWORDS: New technologies; metaphysics; virtual reality; simulation; appearance; potentiality; mediation; construction; Universe; mind; artificial intelligence.

"Los gráficos están geniales, pero el argumento es penoso"

Comentario de un alumno del Profesor Brian Whitworth

Con Ulises

La metafísica es esa disciplina filosófica que trata sobre la realidad en un sentido que va más allá de la física. Intenta entender toda la realidad y todo nuestro conocimiento de la realidad, incluyendo la realidad que la física describe e incluyendo también el propio conocimiento físico de la realidad. Cabe decir que la metafísica quiere entender toda la realidad y todo nuestro conocimiento de la realidad pensando en términos de ciertos principios. ${ }^{2} \mathrm{El}$ empeño metafísico es sumamente ambicioso. La comprensión que busca es total. Pero justo por ello, la metafísica puede llegar de un modo muy directo a la convicción de que entender la realidad y nuestro conocimiento de ella es algo que sólo puede hacerse de manera parcial e inacabada. ${ }^{3}$

Es importante darse cuenta de que únicamente cabe llegar a la conclusión anterior si perseguimos una comprensión total. Si lo que se busca es tan sólo entender

2 A veces se habla de "primeros" principios, otras veces de principios "últimos". Depende del punto de vista. Queremos comenzar nuestro trabajo con estas caracterizaciones clásicas para enfatizar las continuidades entre algunos problemas filosóficos tradicionales y los nuevos problemas planteados por la realidad virtual.

${ }^{3}$ La ontología es otra disciplina estrechamente relacionada con la metafísica. Podemos diferenciar ambas por su relativo alcance y ambiciones. La ontología describiría los rasgos más generales de ciertas partes de la realidad y de nuestro conocimiento de ellas, sin necesariamente aspirar a describir toda la realidad, ni necesariamente aspirar a una comprensión última. Esta distinción es importante. Actualmente, la ontología es un apartado muy importante en áreas como la creacción de lenguajes de programación o el diseño de sistemas expertos. 
una parte de la realidad o una parte de nuestro conocimiento de la realidad, no cabe llegar a la conclusión de que entender toda la realidad, incluyendo nuestro conocimiento de ella, es algo que sólo puede hacerse de manera parcial e inacabada. Podemos expresar la idea que está detrás de estas observaciones de forma muy simple: sólo haciendo metafísica pueden obtenerse conclusiones metafísicas.

En este trabajo vamos a hacer metafísica de la realidad virtual. ${ }^{4}$ Comenzaremos analizando algunos rasgos de lo virtual. Y a continuación, exploraremos en profundidad varias posibilidades de virtualidad para el Universo y para la mente.

\section{REALidAD VIRTUAL Y LENGUAjE}

Pensemos en la siguiente caracterización de la realidad virtual:

Realidad Virtual: Entorno de apariencia real, o de realidad aparente, que, a pesar de provocar una fuerte sensación de inmersión, habitualmente generada mediante tecnologías computacionales, puede no tener la clase de realidad que parece tener.

La realidad virtual nos es familiar en contextos de ficción. Dos ejemplos recientes son la serie de películas de la saga Matrix (la primera en 1999) y la película Desafí total (1990). Otro ejemplo más clásico, pero igual de inquietante, aún sin involucrar el uso de tecnología computacional, es el relato La invención de Morel, de Bioy Casares (1940). Fuera de la ficción, hay actualmente varios tipos de dispositivos creadores de realidad virtual en la modalidad sensorial de la visión. Un entorno de realidad visual con inmersión máxima llevaría esto al extremo, llegando a todas las modalidades sensoriales.

Actualmente, la tecnología computacional está estrechamente relacionada con la realidad virtual. Sin embargo, no es necesaria la tecnología computacional para crear realidad virtual. Hacer que sea necesaria empobrece el concepto. Hay dos razones de peso en contra. Acabamos de mencionar la primera de ellas. Se puede crear realidad virtual sin tecnología computacional. Bastan las tramoyas del teatro. Y basta el lenguaje. El lenguaje es el gran creador de realidad virtual. La otra razón

\footnotetext{
4 Tomamos prestada la expresión "metafísica de la realidad virtual” de Heim (1993). Véase también Heim (1986) y Heim (1998). Asímismo, deben destacarse los análisis de Chalmers (2009) y Chalmers (2017), quien defiende un fuerte realismo respecto a la realidad virtual. Según Chalmers, la realidad virtual no sería una simple ficción, sino un tipo peculiar de genuina realidad. Y la vida en mundos virtuales puede llegar a tener el mismo tipo de valor que la vida en lo que podemos llamar mundo real.
} 
la encontramos en la ciencia. Tal vez todo nuestro mundo macroscópico ordinario no sea sino una especie de inmensa ilusión generada por fenómenos cuánticos. Todo el Universo puede ser una inmensa proyección holográfica generada por algo mucho más básico.

Pero analicemos más de cerca el lenguaje. Considerar que nuestras capacidades de simbolización o conceptualización ligadas al lenguaje son generadoras de realidad virtual enriquece enormemente nuestra perspectiva. Hace que la realidad virtual no sea un fenómeno completamente nuevo. Cabe decir que la realidad virtual nos acompaña desde el mismo comienzo de la humanidad. Toda la realidad que nos circunda rápidamente se convirtió en una "realidad ampliada" con la aparición del lenguaje. ${ }^{5} \mathrm{Y}$ desde esos inicios, nuestra realidad no ha dejado de cobrar nuevos relieves. ${ }^{6}$

Desde sus mismos orígenes, el lenguaje se convirtió en una poderosa fuente de realidad virtual. Y se mezcló de manera inextricable con todos nuestros entornos reales. El contenido conceptual de toda experiencia característicamente humana y la carga teórica de toda observación son los últimos peldaños de esa escalera que comienza con un pie en la realidad y con otro pie en la fabulación simbólica.

\section{Platón, Aristóteles, Descartes y Kant}

Hay cuatro formas muy distintas de entender lo virtual (la naturaleza de lo virtual, la existencia de lo virtual). Todas ellas son importantes. Y todas ellas han sido desarrolladas con bastante detalle en el contexto de nuestra filosofía.

\section{Platón: Lo virtual como apariencia}

Para Platón, la auténtica realidad es ideal. Consiste en lo que las cosas deben ser. Suele hablarse de mundo de las ideas frente al mundo sensible. Las ideas son entidades normativas. Son los ideales de las cosas. Son los patrones, los moldes, las

\footnotetext{
5 Podemos entender la "realidad ampliada" como un entorno real mezclado con elementos virtuales creando realidades mixtas en tiempo real, o cercano a un tiempo real.

${ }^{6}$ Relieves formados por colecciones de aspectos y de sentidos actuando literalmente como formas constituyentes (platónicas, aristotélicas o kantianas, aquí no importaría mucho). Esta manera de ver y entender la realidad virtual se pierde, o al menos se difumina, en enfoques obsesionados por las posibilidades que ofrece la actual tecnología computacional. Como ejemplo de esto, véase Chalmers (2009) y Chalmers (2017). Su fuerte realismo respecto a la realidad virtual ganaría plausibilidad, convirtiéndose casi en trivialidad, en posición por defecto, si se asumiera que el lenguaje basta para crear virtualidad y que la existencia de realidad virtual no depende esencialmente de la actual tecnología computacional.
} 
normas de las cosas. Cómo debe ser un árbol, una mesa, un ser humano, la virtud, el conocimiento, etc. El mundo sensible es el mundo de las cosas particulares y concretas, cosas en continuo cambio. Y este mundo es simplemente apariencia. Es mera "pretensión". Todos los árboles particulares y concretos pretender ser el árbol ideal, todas las mesas pretenden ser la mesa ideal, todos los seres humanos el ser humano ideal, todas las acciones virtuosas aspiran a encarnar la virtud ideal, todos los conocimientos el conocimiento ideal, etc.

Así es como Platón conceptualiza la distinción apariencia/realidad. Y su manera de trazar esta distinción nos permite identificar lo virtual con las apariencias.

Lo virtual es apariencia. Y se opone a lo auténticamente real. Lo auténticamente real, la realidad real podemos decir, es ideal. Es un patrón, un molde, una norma. Y todo lo no ideal es simplemente apariencia. Como hemos dicho, mera pretensión.

La figura del Demiurgo es muy importante en Platón. Necesita explicar por qué existen las apariencias. Necesita explicar por qué no existe tan sólo la auténtica realidad de las ideas. Sin esta explicación, la imagen que se ofrece resulta insuficiente. Pero el problema es que ni las ideas ni las apariencias pueden ofrecer tal explicación. Por un lado, apelar a las ideas no basta para explicar por qué existen las apariencias. Por otro lado, apelar a las apariencias para explicar por qué existen las apariencias sería circular. Platón recurre a una extraña entidad casi divina: el Demiurgo. El Demiurgo no pertenece ni al mundo ideal ni a las meras apariencias. Pero crea el mundo sensible, las apariencias, tomando como referencia las ideas. Igual que un artesano elabora sus productos pensando en cierto patrón, utilizando ciertos moldes, intentando ajustarse a cierta norma. ${ }^{7}$

Así pues, lo virtual puede entenderse como apariencia. Y el modelo sería el de un artesano que en sus producciones imita, con mejor o peor fortuna, cierto patrón.

\section{Aristóteles: Lo virtual como potencialidad}

Aristóteles sólo acepta un mundo, no dos. Y en ese único mundo hay sustancias con un alto grado de independencia y desarrollo autónomo. La realidad

\footnotetext{
7 ¿Puede trazarse también respecto al Demiurgo una distinción apariencia/realidad? Si la respuesta es positiva, nos vemos envueltos en un problema de regreso (distinto, por cierto, del clásico problema de regreso que introduce el argumento del "tercer hombre"). Sin embargo, al tratarse de un ser "casi divino", la respuesta podría ser negativa. Con el Demiurgo, no podría distinguirse apariencia y realidad. Aunque esto hace surgir otra cuestión. ¿̉Hay entonces algo más que ideas y apariencias? Platón es de esta opinión en muchos textos, por ejemplo, en el Timeo. Esto nos fuerza a cambiar bastante nuestras interpretaciones habituales de Platón.
} 
está constituida por sustancias particulares y concretas. Las sustancias tienen un modo de ser esencial, sin el cual dejarían de ser las sustancias que justamente son. Y también tienen modos de ser accidentales. Además, las sustancias están constituidas por una materia y una forma. Y su existencia combina potencialidad y actualidad. El mundo de Aristóteles está lleno de disposiciones y procesos. Pero nada de esto es separable de las sustancias particulares y concretas que realmente existen. Separadas de tales sustancias particulares y concretas (las llamadas "sustancias primeras") la esencia y los accidentes, la materia y la forma, la potencialidad y la actualidad, son tan sólo abstracciones.

Aristóteles nos ofrece otra forma de conceptualizar lo virtual. Podemos identificarlo con la potencialidad de las sustancias. Este es otro de los significados nucleares que hoy en día sigue teniendo el término "virtual". Y así hablamos con frecuencia de virtualidad como sinónimo de potencialidad.

Pero, como ya hemos indicado, las potencialidades no pueden separarse de las sustancias. Si lo hacemos, lo único que tenemos es una abstracción. En otras palabras, las puras potencialidades no son reales.

El Demiurgo estaría ahora, por decirlo así, dentro de cada sustancia. Sería una fuerza dinamizadora interna a cada sustancia. Y esa fuerza se manifiesta como actividad. Lo que existe en potencia, pasa a existir en acto. ${ }^{8}$

Al hilo de este planteamiento, lo virtual puede entenderse como potencialidad. Pero debemos insistir en que esta potencialidad, si se separa de las sustancias particulares y concretas que la poseen, se convierte en una simple abstracción. Se convierte en algo que ya no es real.

No lo hemos dicho, pero es bien sabido. Para Aristóteles, el modelo de lo que son las sustancias (esas "sustancias primeras") lo constituyen las entidades orgánicas: plantas y animales. Y sobre todo, las personas.

\section{Descartes: Lo virtual como mediación}

La búsqueda cartesiana de la certeza, eliminando toda duda, por pequeña que sea, nos ofrece otro significado para lo virtual. En su camino hacia algún tipo de certeza, Descartes considera la posibilidad de que un demonio maligno

\footnotetext{
8 Una cuestión importante: Esa fuerza dinamizadora, interna a cada sustancia, que llega a definir cada sustancia particular y concreta, ¿debe aceptarse como un componente último de la realidad? Esto estaría muy cerca de una posición que considerara que las unidades más básicas de la realidad son los procesos.
} 
esté engañando nuestros sentidos ${ }^{9}$, de manera que todo el contenido de nuestras experiencias no merezca más crédito que el crédito que puede tener el contenido de nuestros sueños. En tal situación, persistir en nuestras creencias perceptivas nos hundiría cada vez más en el error. Y esto se transmitiría a todo nuestro presunto conocimiento, desde la experiencia ordinaria hasta la ciencia más elaborada. Aunque la realidad fuera de hecho como parece ser, careceríamos de justificación para creer que es así. Y estaríamos engañándonos al no considerar que un demonio maligno puede estar engañándonos.

¿Cómo descartar esta posibilidad? ¿Cómo descartar una hipótesis como la de este genio maligno? Se nos ha dicho muchas veces que gran parte de la filosofía moderna comienza justamente aquí. Pero no queremos discutir ahora estas cuestiones. ${ }^{10}$ Tan sólo queremos poner de relieve otro significado, por cierto, también muy frecuente, asociado a lo virtual.

Lo virtual puede entenderse como mediación. Y lo que hace que esta mediación llegue a ser engañosa es su posible desconexión con la realidad, o siendo más precisos su posible conexión no adecuada con la realidad. Esto es lo que produce el engaño, y el autoengaño, o cuando menos la posibilidad de un error difícilmente detectable.

El Demiurgo se ha convertido ahora en demonio o genio maligno. Y cualquier mediación no adecuada puede desempeñar su papel. ${ }^{11}$ Los modelos son el sueño, las ilusiones, las alucinaciones y las delusiones.

9 Tal vez su engaño incluso llegue más dentro de nosotros, y afecte a nuestros razonamientos, nuestra memoria, etc. La realidad virtual generada también puede ser "interna" a los sujetos, en algún sentido más profundo que el sentido en el que son internas nuestras experiencias de un "mundo externo". ¿'Tiene algún límite esta posible generación de engaño? Descartes creía que sí. Dejaremos también al margen este tema.

10 Aumentar el número de nuestras creencias verdaderas sin considerar esa hipótesis aumentaría nuestro conocimiento animal, pero no nos acercaría al tipo de conocimiento más propiamente humano, un conocimiento reflexivo que no sólo consiste en tener un conocimiento de primer orden que podemos llamar "conocimiento animal", sino también en tener conocimiento acerca de porqué se conoce. Véase al respecto el novedoso análisis de la epistemología cartesiana, concretamente del clásico círculo cartesiano, ofrecido por Sosa (2009).

11 Vuelve a surgir ante nosotros una pregunta estructuralmente similar a la que formulábamos respecto al Demiurgo platónico. ¿Es ese demonio maligno sustancia pensante o sustancia extensa? Desde luego, no podría ser Dios (ese tercer tipo de sustancia), al menos no para Descartes. Y podría ser tanto sustancia pensante como sustancia extensa, y también ambas cosas a la vez. Sin duda, puede haber muchos demonios malignos, de muchos tipos. 


\section{Kant: Lo virtual como construcción}

En Kant encontramos una última manera de entender lo virtual. No menos importante que las otras tres. Para Kant, como para Descartes, y en contraste con Platón y Aristóteles, las ideas están en la mente. ${ }^{12}$ Pero a diferencia de Descartes, la mente impone las ideas sobre la realidad obteniendo hechos de experiencia y contenidos de conocimiento. Las ideas son como moldes para hacer galletas. La masa que se moldea es la realidad en sí misma. No es más que una $\mathrm{X}$ incognoscible. $\mathrm{Y}$ la realidad que experimentamos y conocemos es una construcción del sujeto a partir de esa X. A lo primero, Kant lo llama "noúmeno". A lo segundo, "fenómeno".

La construcción no es, para Kant, arbitraria. No podemos construir la realidad como queramos. Hay constricciones conceptuales y constricciones empíricas. Sea como sea, el peligro de la arbitrariedad ha estado siempre latente en el planteamiento kantiano. Y es muy fácil identificar directamente lo virtual con lo construido, con lo moldeado por las ideas. Y también, identificar la auténtica realidad con esa realidad en sí misma únicamente representable como una X. Lo virtual es fenómeno. Y en último término, la única realidad, digamos, real es la masa nouménica primigenia.

El modelo es ahora el de un supremo constructor. Tan constructor como para modificar y convertir en construcción todo aquello que mira o toca, como Midas convertía todo en oro. Pero las construcciones no son arbitrarias. Acaban derivándose de nuestra condición de seres racionales. $\mathrm{O}$ al menos, eso se postula.

¿En qué se ha convertido ahora el Demiurgo? El Demiurgo es un "yo" que construye todos los fenómenos moldeándolos en un espacio-tiempo y dotándoles de todos los rasgos generales que podemos encontrar en el mundo tal como lo experimentamos y conocemos. ${ }^{13}$

\footnotetext{
12 Bueno, están en la mente en algún sentido. De manera más precisa, están en un sentido "trascendental". Son condiciones de posibilidad. Y por cierto, debe quedar claro que estamos usando el término "idea"en un sentido muy genérico, como algo equivalente a "concepto". No estamos refiriendonos aquí a las "ideas de la razón".

13 El relativismo siempre ha sido una amenaza contante para los planteamientos de tipo kantiano. ¿Cómo librarse de él? Kant lo hacía postulando la universalidad de la construcción para todo ser racional. Otra forma de expresarlo: el "yo" que construye el mundo experimentado y conocido es un yo trascendental. Sobre la gran importancia filosófica del relativismo, véase Hales (2006).
} 
En nuestro pequeño recorrido, hemos encontrado cuatro importantes significados para lo virtual. Lo virtual puede entenderse como:

1. Apariencia

2. Potencialidad

3. Mediación

4. Construcción

Debe ponerse de relieve que ninguno de estos cuatro significados es concluyentemente negativo. Las apariencias pueden ser "meras" apariencias, la potencialidad puede ser únicamente una "abstracción", la mediación puede ser "engañosa" y la construcción puede ser "arbitraria". Sin embargo, esto sólo es una cara de la moneda. Y la otra cara puede llegar a ser altamente positiva. Las apariencias pueden ser aspectos bajo los cuales la propia realidad se nos haga accesible, las potencialidades también pueden ser constituyentes esenciales de la realidad, la mediación puede establecer vínculos adecuados y la construcción puede ser perfectamente no arbitraria.

¿Cómo integrar los anteriores cuatro aspectos? ¿Cómo obtener una concepción llena de contenido y equilibrada de la realidad virtual? A continuación, señalaremos a muy grandes rasgos un camino tentativo. Será un camino arriesgado, pero también prometedor.

De manera concentrada, dicho camino es el siguiente. En primer lugar, la normatividad presente en el planteamiento de Platón debería preservarse. Sin normatividad, perdemos demasiado. Pero no debe preservarse esa normatividad en un sentido trascendente, sino inmanente. Debería ser una normatividad interna a las propias apariencias, en un sentido muy cercano al que encontramos en Aristóteles. Y esta inmanencia debe depurarse de sustancialismo. Debe volverse más procesual. Con ello, la mediación presente en Descartes tendría el camino abierto para reencontrar la deseada adecuación. Y las construcciones kantianas podrían ganar universalidad. No a través de una siempre arriesgada postulación trascendental, sino en procesos abiertos de convergencia.

Existe, pues, un camino. Y merece la pena explorarlo. Los cuatro significados que hemos encontrado para lo virtual son perfectamente compatibles y mutuamente enriquecedores. La realidad virtual es apariencia, potencialidad, mediación y construcción. Y como hemos dicho, esos significados no sólo tienen connotaciones negativas. También pueden ofrecer una amable cara positiva. ${ }^{14}$

\footnotetext{
14 Sobre las conexiones de la noción de realidad virtual con las nociones de realidad aumentada, inteligencia artificial, humanidad mejorada e Internet de las cosas, véase Liz (2020).
} 


\section{CASOS LÍMITE DE REALIDAD VIRTUAL: EL UNIVERSO Y LA MENTE}

Hay dos casos límite de realidad virtual: el Universo y la mente. Obviamente, los dos son muy importantes. Pero, sobre todo, es importante ser sensible a lo que en cada caso implicaría afirmar que podemos estar ante realidades virtuales.

El Universo es la objetividad más extrema. No hay objetividad más allá del Universo. Simplemente, cualquier cosa que supuestamente estuviera más allá del Universo debería acabar quedando incluida en él. Cabe decir que incluso los universos paralelos sobre los que podemos pensar y hacer teorías serían parte del gran Universo (o Multiverso). ¿Podría todo el Universo no ser más que una realidad virtual? ¿Podría no ser más que apariencia, potencialidad, mediación y construcción? Esto implicaría que cada supuesta apariencia no sería más que un aspecto de otra apariencia, cada potencialidad algo basado en otras potencialidades, cada mediación un camino hacia otra mediación, y cada construcción algo hecho con los materiales de construcciones previas. ¿Hay algún problema en que el Universo sea así, siempre así, en cualquier lugar y momento así?

Debemos tener en cuenta un detalle que no puede distraernos. Aunque todo el Universo no fuera más que realidad virtual, aún así, como ya hemos dicho, las apariencias podrían ser aspectos de la propia realidad, las potencialidades también podrían ser partes constitutivas de la realidad, las mediaciones podrían ser adecuadas y las construcciones no arbitrarias. El problema, si lo hay, no está aquí.

Cambiemos los focos. Consideremos ahora la mente. ${ }^{15}$ Es la subjetividad más extrema. La mente es la fuente última de lo subjetivo, su referencia constante, el paradigma. ¿Podría la mente no ser más que realidad virtual? ¿Podría no ser más que apariencia, potencialidad, mediación y construcción? Desde luego, si la mente se integrara en el Universo y todo en él fuera realidad virtual, entonces también habría de serlo la mente. Pero con independencia de esto, en un Universo que no fuese sólo realidad virtual, ¿podría serlo la mente? Lo que estamos preguntando es sin duda metafísicamente interesante. Se trata de que puedan existir "islas" de realidad virtual, algo así como "islas mentales", incluso en un Universo en el que no todo sea realidad virtual.

\footnotetext{
15 No deja de ser asombrosa nuestra capacidad para referirnos al Universo, en singular y con mayúscula. Pero no es menos asombroso que no hagamos lo mismo con la mente. Al decir "el Universo", excluimos estar hablando tan sólo de un universo. Al decir "la mente", excluimos estar hablando de la Mente, de una única y singular mente. ¿Por qué? ¿Qué contingencias nos han llevado a estas peculiares orientaciones semánticas y sintácticas?
} 
¿Hay localizaciones espaciotemporales en las que adscribimos apariencias que nunca son aspectos mas que de otras cosas que también son apariencias, potencialidades que nunca están basadas mas que en otras potencialidades, mediaciones que siempre conectan otras mediaciones, construcciones sobre otras construcciones? Si la respuesta es afirmativa, tendríamos "islas" de realidad virtual. Y nuestras mentes constituidas únicamente como realidades virtuales serían "islas" de ese tipo. ${ }^{16}$

Estos dos casos límite de realidad virtual, el Universo y la mente, tienen algo más en común. En ambos casos parece que debería haber algo no virtual como "base", un sustrato no virtual, algo no virtual produciendo esas virtualidades. Y en ambos casos, el argumento es muy simple. Consiste en afirmar que no todo puede ser así porque no podemos "entender" que sea así. No parece poder ser "todo" realidad virtual ni en la objetividad más extrema ni, tampoco, en la subjetividad más extrema.

Todos los demás casos entre la objetividad extrema del universo y la subjetividad extrema de la mente pueden ser sin demasiados problemas casos de realidad virtual. Los árboles que vemos a través de la ventana pueden ser realidades virtuales. Igual que nuestros sueños o los recuerdos del viaje que hicimos a París. Esas cosas sí son perfectamente asumibles como realidad virtual. Pero no "todo" el Universo, ni tampoco "toda" la mente.

Es más, no se puede componer con "trozos virtuales" todo el Universo. Ni tampoco toda la mente. Esto tampoco funciona. Nuestras intuiciones espontáneas son muy fuertes al respecto. No se consigue hacer que todo el Universo "pueda ser virtual" juntando, o sumando, o agregando, todas sus partes que efectivamente puedan ser virtuales, y ciertamente son todas. Exactamente lo mismo cabe decir de la mente. Por separado, cualquier parte puede ser virtual porque otras partes no lo son. Y si juntamos, o sumamos, o agregamos, todas esas realidades virtuales, desaparece ese soporte, esa base que parece imprescindible.

${ }^{16}$ Es obvia la sintonía de esto con la noción de "mónada", tematizada por Leibniz sobre todo en su Monadología. En nuestras "islas mentales" hay tanto elementos representacionales como elementos motivacionales dinamizadores. Y las adscripciones de localización espaciotemporal física a una "isla mental", a un "yo" personal podemos decir también, no implican ninguna distribución de sus diferentes componentes en diferentes regiones espaciotemporales físicas. Es como si la localización de una "isla mental" en el espacio-tiempo físico no admitiera más descomposición espaciotemporal física, como si se tratara efectivamente de un "punto". 
Pero debemos ir al fondo de la cuestión. Tenemos que preguntar, ¿por qué no "entendemos" bien que todo el Universo o toda la mente puedan ser realidades virtuales? A continuación, examinaremos más de cerca esta pregunta. ${ }^{17}$

\section{4. ¿PODRÍA TODO NUESTRO UNIVERSO SER UNA REALIDAd VIRTUAL?}

¿Podría todo nuestro Universo ser una inmensa realidad virtual? Debemos considerar dos posibilidades muy discutidas actualmente: ${ }^{18}$

P-1: El Universo como siendo una proyección de la realidad cuántica

Tendríamos aquí un cierto realismo cuántico combinado con la tesis de que todo lo demás es una especie de gran proyección. ¿Dónde estaríamos nosotros, nuestra mente? ¿Sería parte de la realidad cuántica, una parte tal vez crucial? ¿Quedaría subsumida nuestra mente en esa gran proyección? Las dos opciones están abiertas.

P-2: El Universo como la ejecución de un gran programa informático

El Universo sería aquí una gran simulación computacional. Y nosotros mismos seríamos o bien parte de lo que produce esa simulación, o bien parte de la misma simulación. También se abren aquí estas dos opciones.

Otras épocas, otras culturas, han considerado, o consideran, muy diferentes posibilidades para una realidad virtual total. Pero centrémonos en estas dos. La mayoría de nuestros planteamientos y conclusiones serían generalizables. Dejemos también al margen, de momento, dónde situar nuestra mente en esas dos posibilidades. Es decir, dejemos por ahora al margen las dos opciones que se

${ }_{17}$ Nuestra pregunta puede mezclarse con preguntas epistemológicas de tono escéptico. ¿Es posible que todo a nuestro alrededor no sea más que una gran alucinación? ¿Es también posible que hasta lo que nos parece más obvio y evidente no sea más que una confusión desordenada de sensaciones? No estamos tratando aquí estas preguntas. De todos modos, frente al escepticismo tampoco basta con "agregar". La suma de todas las posibles dudas parciales no arroja como resultado una duda total.

${ }_{18} \mathrm{La}$ concepción del universo como holograma generado por una realidad más básica, por ejemplo, de tipo cuántico, se ajustaría a P-1. La concepción del universo como una gigantesca simulación informática se ajustaría a P-2. No daremos ninguna referencia a autores concretos. Pero es sencillo encontrarlas. El origen de estas dos posibilidades se encuentra en la ciencia. Pero tienen un enorme peso cultural y artístico fuera de la ciencia. Y desde hace años actúan con fuerza en nuestro imaginario colectivo. 
abren en cada una de las anteriores posibilidades. En cualquier caso, hay un grave problema que es común a P-1 y P-2.

Hay un grave problema en la idea de que una realidad virtual total pueda ser generada de alguna de esas dos formas, o de otras similares. El problema consiste en que, si la realidad virtual es total, si abarca absolutamente todo, entonces su producción ha de ser "de un tipo constitutivo completamente diferente" a cualquier cosa que podamos llegar a encontrar en lo producido. No podrá ser una realidad virtual generada de ningún modo habitual que nosotros podamos llegar a experimentar o conocer.

Hay una profunda tensión entre afirmar que toda la realidad puede ser una realidad virtual y explicar, o siquiera entender a grandes rasgos, cómo podría ser así. Y a este respecto, incluso la paradójica realidad cuántica y la ejecución de un enormemente complejo programa informático podrían contar como "modos habituales" de generar una realidad virtual. Pueden ser considerados modos habituales justamente en la medida en que estas cosas también pueden ser consideradas realidades virtuales.

Es fácil encontrar ejemplos de lo que estamos diciendo. La saga Matrix (primer estreno, 1999) intenta mostrarnos una realidad virtual total. Pero sólo lo intenta. Porque en ella se pone de manifiesto la anterior tensión. Cuando algunos de los protagonistas “despiertan”, liberándose de la conexión al supercomputador que es Matrix, se encuentran con una realidad que es completamente similar a la realidad que vivían estando conectados a Matrix. Podemos decir que la realidad en la que despiertan es del mismo "tipo constitutivo" que la realidad virtual en la que estaban inmersos. Claro, no es similar en cuanto a las cosas particulares y concretas que se encuentran (al despertar, todo es más ruinoso, sucio y desolado), pero sí en cuanto a las "clases constitutivas de cosas" que puede haber. Y el punto crucial es que una realidad virtual como ésta, una simulación de este tipo, no puede ser total.

Si se trata de una simulación total, entonces lo que haya "fuera" de Matrix, si es que hay algo, tiene que ser completamente diferente de todo lo que podamos encontrar "dentro" de Matrix. Tiene que ser de un "tipo constitutivo" completamente distinto. ${ }^{19}$

19 Como ya hemos dicho, esto admitiría una generalización. Pueden decirse las mismas cosas, por ejemplo, de la posibilidad de que todo sea un sueño. O de la posibilidad de que todo, absolutamente todo, sea una construcción. O de la posibilidad de estar siendo completamente engañados. Y un largo etc. 
5. TRES TESIS SOBRE LO VIRTUAL

A continuación, vamos a introducir tres importantes tesis sobre la realidad virtual. Las utilizaremos en el resto de nuestro trabajo. Ya hemos señalado la primera. Se trata de lo siguiente:

\section{T-1: Una realidad virtual total ha de ser generada, o producida, o constituida, de manera completamente no habitual desde nuestros puntos de vista}

Si la realidad virtual es total, entonces no puede ser generada, o producida, o más en general constituida, a través de ningún mecanismo o medio habitual. También podemos decir que una realidad virtual total ha de ser generada, o producida, o constituida, de manera que no quede implicado nada que pudiéramos llegar a considerar una realidad virtual.

"Realidad virtual" y "simulación" pueden utilizarse aquí como sinónimos. Como hemos dicho, cabe expresar T-1 diciendo que una simulación total ha de ser una simulación en cuya generación o producción no quepa encontrar nada que podamos "llegar a considerar" una simulación. Por tanto, si se trata de una simulación total, no puede ser producida de ninguna manera habitual. En otras palabras, si es una simulación generada por algún procedimiento habitual, entonces no podrá ser considerada total. Habrá siempre espacio para la sospecha de que, después de todo, no se trata de una simulación total. Dicho de otro modo, siempre quedará abierta la sospecha de que realmente existen (como realidades no virtuales) cosas como las que parecen existir y de que en base a esas cosas se generan, producen o constituyen (como realidades virtuales) apariencias de cosas como las que parecen existir.

\section{T-2: Lo virtual, en todos sus sentidos, se resiste a admitir una distinción apariencia/realidad}

No podemos decir que algo parece ser una cierta realidad virtual pero no lo es en realidad. Respecto a la realidad virtual, no se aplica la distinción apariencia/ realidad. Si algo parece ser una cierta realidad virtual, entonces realmente lo es. Si algo parece ser un árbol virtual, entonces realmente es un árbol virtual. Para la realidad virtual, parecer implica ser.

Esta tesis se aplica a lo virtual en los cuatro sentidos que hemos recogido. Comencemos con el sentido platónico en el que virtual significaba apariencia. La apariencia de una apariencia es una apariencia. De igual modo, si algo parece una simulación, entonces realmente es una simulación. Simular una simulación es simular. 
También puede decirse lo mismo de ese sentido aristotélico de lo virtual que se vinculaba estrechamente a potencialidad. Introduzcamos un nuevo concepto.

Digamos que la disposición "particularmente vinculada a una cierta manifestación" es la disposición consistente en poder tener esa manifestación y ninguna otra.

Una disposición particularmente vinculada a una manifestación es simplemente un poder manifestarse exclusivamente de un cierto modo máximamente específico. Las simulaciones también son un modo de manifestarse. Y cabe asumir que ha de existir siempre una disposición particular vinculada a ese modo de manifestarse, un poder manifestarse de esa manera peculiar. La introducción de la anterior noción tiene una motivación muy intuitiva. Si algo ocurre, podemos asumir que existe una disposición particularmente vinculada a que ocurra justamente como ocurre.

Sin embargo, una cosa es que detectemos que ocurre algo y otra que ocurra del modo como ocurre. Las disposiciones particularmente vinculadas a una cierta manifestación no son simplemente lo que "constatamos" que ocurre. Hablar de ellas no es simplemente otra forma de hablar de lo que nos es manifiesto. Lo que ocurre fácilmente puede "pasar desapercibido". Las manifestaciones de una cierta disposición pueden no ser detectadas. Y también pueden ocurrir en otros tiempos y lugares, pueden tener lugar en "otros contextos" diferentes. Ni el que una manifestación pase desapercibida ni el que pueda tener lugar en otros contextos implica que deba cambiar la disposición particularmente vinculada a tal manifestación.

Las disposiciones particularmente vinculadas a una cierta manifestación consisten en el poder ocurrir justamente lo que está ocurriendo, ni más ni menos. $Y$ por ello, parecer que se tiene una disposición particularmente vinculada es tenerla realmente. No hay ningún espacio lógico para la duda. ${ }^{20}$

Puede ser muy difícil identificar disposiciones particularmente vinculadas a una manifestación. Sobre todo, como decimos, porque las manifestaciones fácilmente pueden "pasar desapercibidas". Y también porque pueden "ocurrir" en otros tiempos y lugares, darse en otros contextos diferentes. Realmente, es difícil identificar de manera precisa un modo de manifestarse. Pero no estamos tratando estas cuestiones. Lo que queremos subrayar es que, respecto a las disposiciones particularmente vinculadas, no habría tampoco ninguna distinción apariencia/

${ }^{20}$ Las disposiciones particularmente vinculadas no admiten ninguna generalización. No tienen proyección en ninguna dimensión modal diferente de lo que de hecho ocurre. 
realidad. Si algo pareciera completamente ser una disposición particularmente vinculada a cierta manifestación, entonces sería realmente la disposición particularmente vinculada a tener esa manifestación. Y también podemos decir que, si algo simula completamente una cierta disposición particularmente vinculada a determinado modo de manifestarse, entonces realmente será esa disposición particularmente vinculada.

En definitiva, la realidad virtual tanto de las simulaciones como de las disposiciones particularmente vinculadas a ellas implica su realidad real. ¿Sobre qué se aplica, entonces, la distinción apariencia/realidad? La respuesta a esta pregunta muestra algo sumamente importante. La distinción apariencia/realidad se aplica a todo lo que "pretende" no ser tan sólo realidad virtual. Según lo que vamos viendo, se aplicaría a lo que "pretende" no ser tan sólo ni una simulación ni una disposición particularmente vinculada. ¿Y qué puede existir que no sea nada de esto? Pues la mayoría de las cosas y hechos que creemos percibir y la mayoría de las cosas y hechos que creemos pueblan el mundo: la mesa sobre la que creo estar escribiendo, el árbol que creo ver a través de la ventana, los electrones que creo detectar atravesando una particular rendija, que esté realmente lloviendo en París, que realmente mi vecina no pare de estornudar, que realmente se forme cierto agujero negro en algún lugar del universo, etc. La distinción apariencia/ realidad se aplica a todo aquello que o bien pretenda ser no sólo una manifestación o bien a lo que pretenda ser no sólo una disposición particularmente vinculada.

La distinción apariencia/realidad se aplica siempre "a una pretensión". Y mientras las simulaciones y las disposiciones particularmente vinculadas a una cierta manifestación no pretendan ser otra cosa, sobre ellas no podremos decir que aunque parezcan ser simulaciones y disposiciones particularmente vinculadas, realmente no lo son. ${ }^{21}$

21 Dos manifestaciones pueden tener exactamente la misma apariencia, pero ser realmente manifestaciones distintas por proceder de diferentes disposiciones particularmente no-vinculadas. Cuando las simulaciones se consideran manifestaciones de ciertas disposiciones particularmente no-vinculadas, tenemos esto. Sin embargo, no hay "mera simulación" de la disposición particularmente vinculada. En términos de "poderes causales", simular perfectamente un poder causal particularmente vinculado es tenerlo realmente. Esto ofrece una objeción de peso a las estrategias empleadas por John Searle para criticar la inteligencia artificial en sentido fuerte. Véase Searle (1980), Searle (1985) y Searle (1990). Por supuesto que puede haber meras simulaciones de las manifestaciones (conversacionales, conductuales) de una mente. Incluso una simulación perfecta de esas manifestaciones puede ser tan sólo una "mera simulación". Pero respecto a la capacidad de tener justamente esas manifestaciones, 
Según decíamos, hay cuatro significados generales que la filosofía ha asociado a lo virtual. Lo virtual es apariencia, potencialidad, mediación y construcción. Cada uno de esos significados ha sido resaltado por un autor particular. Hemos hablado de Platón, de Aristóteles, de Descartes y de Kant. Está claro que sobre las propias apariencias la distinción apariencia/realidad no funciona. Y que no hay grandes diferencias entre las apariencias y las simulaciones. Respecto a las simulaciones, consideradas en sí mismas, tampoco funciona la distinción apariencia/realidad. Puede sorprender que lo mismo ocurra con el segundo significado asociado a lo virtual, lo virtual como potencialidad. Pero en un importante sentido así es. Las disposiciones particularmente vinculadas no se someten a la distinción apariencia/ realidad. Parecer tener una cierta disposición particularmente vinculada es tenerla realmente. Y parecer poder tener ciertas manifestaciones particulares, acaso ciertos efectos causales particulares, es poder tener esas manifestaciones o efectos particulares. Tampoco existe aquí espacio para una distinción apariencia/realidad. Si no se tuviera realmente una cierta disposición particularmente vinculada, entonces no podría parecer que se tiene. La cercanía entre la disposición y su manifestación es aquí tan estrecha que parecer que se tiene la disposición es tenerla realmente. No suele tenerse en cuenta este rasgo de las disposiciones. Pero es tremendamente importante. $\mathrm{Y}$ afecta crucialmente a muchos argumentos filosóficos. $^{22}$

¿Qué decir de los otros dos significados de lo virtual? ¿Qué decir de las mediaciones y de las construcciones? Sin duda, estos significados añaden aspectos nuevos. Sin embargo, lo que las mediaciones y las construcciones tienen de simulación y de potencialidad particularmente vinculada hace que tampoco respecto a ellas tenga sentido la distinción apariencia/realidad. Algo que parece completamente una mediación es realmente una mediación. Y algo que parece completamente una construcción también es realmente una construcción, como las casas y castillos simulados de Disneylandia. Sí, mi hijo siempre dice que allí se podría vivir. Que podríamos vivir en Disneylandia. Y realmente se podría.

De cualquier forma, T-2 es aplicable a todo lo virtual. Y seguramente también sea aplicable a otros miembros de la misma familia que las simulaciones y las

respecto a esa disposición particularmente vinculada, respecto al poder causal particularmente vinculado, ya no caben las "meras simulaciones". Simular un poder causal particularmente vinculado sí es duplicarlo.

22 Como hemos visto, afecta de manera muy especial a los argumentos de Searle mencionados en la nota anterior. 
disposiciones particularmente vinculadas. Pero debemos dejar aquí pendiente esta cuestión. Vayamos a nuestra tercera tesis sobre la realidad virtual.

\section{T-3: La posibilidad de que todo el Universo sea una realidad virtual total nos obliga a asumir la verdad de la inteligencia artificial en sentido fuerte}

La inteligencia artificial en sentido débil simularía procesos que en nosotros requieren mente, sobre todo procesos computacionales. La inteligencia artificial en sentido fuerte afirmaría que así, mediante ciertos procesos computacionales, es posible construir mentes genuinas de manera artificial. ¿Cómo se justificaría nuestra tesis T-3? Con todo el universo convertido en una realidad virtual, parecerían existir dos opciones para lo que sea nuestra mente. Ya las hemos insinuado. Llamémoslas ahora opción A y opción B:

Opción A: Podríamos ser parte de esa realidad virtual total. Nosotros mismos no seríamos más que una simulación. Y nuestra propia mente sería también una simulación.

Opción B: Podríamos no formar parte de esa gran realidad virtual, sino de lo que la produce, o genera, o constituye. Nuestra mente no sería una simulación sino parte de lo que está produciendo, o generando, o constituyendo, la simulación.

Veamos cómo afectan estas dos opciones a la verdad de la inteligencia artificial en sentido fuerte. Comencemos con la opción A. Si nosotros mismos somos una simulación, si nuestra mente es una simulación, entonces por T-2 simular esa simulación sería también una simulación de lo mismo. Y esto haría necesariamente verdadera la inteligencia artificial en sentido fuerte.

Vayamos ahora a la opción B. Aquí interviene crucialmente T-1. Pues si es correcta dicha tesis, entonces no podremos considerar en serio la opción $\mathrm{B}$. Ni siquiera podemos literalmente entenderla. Aunque nos parezca entenderla, o aunque supongamos entenderla, no podemos realmente entenderla.

Entender algo sí está entre esas cosas sobre las que podemos hacer una distinción apariencia/realidad. Entender algo sí pretende ser algo más que "parecer que se entiende". Y también algo más que la disposición particularmente vinculada a ese parecer que se entiende. Y el caso es que, si T-1 es una tesis correcta, entonces no podremos realmente entender lo que la opción B pretende decir.

No podemos entender la opción B porque entenderla implica considerar que nosotros mismos podemos estar fuera de todos nuestros puntos de vista. $\mathrm{Al}$ 
pretender entender la opción B hacemos que la naturaleza de nuestra mente sea para nosotros algo completamente inaccesible..$^{23}$ ¿Qué implica esto respecto a la inteligencia artificial en sentido fuerte? Si no podemos entender cómo nuestra mente podría no ser parte de un Universo completamente virtual, entonces deberemos aceptar que lo que queda, esto es, lo que habitualmente llamamos "nuestra mente", es una simulación. Y, de acuerdo, a T-2, alguna clase de simulación de esa mente habitual deberá poder ser considerada asímismo una mente. Puede que ahora no baste una simulación computacional, puede que se requiera una simulación más biológica, o también una simulación de los entornos pertinentes, pero alguna simulación ha de poder ser considerada como siendo capaz de producir de manera artificial mentes como la nuestra.

En definitiva, si las únicas opciones son $\mathrm{A}$ y $\mathrm{B}$, tendremos que asumir la verdad de la inteligencia artificial en sentido fuerte. La opción A la implica directamente. Y simplemente, no entenderíamos la opción B. No podríamos entenderla. Y lo que queda hará que nuestra mente sea una simulación. Si todo el Universo es una inmensa realidad virtual, tanto la opción A como la opción B nos llevan a asumir la verdad de la inteligencia artificial en sentido fuerte.

\section{6. ¿Y SI NUESTRO UNIVERSO FUERA UNA REALIDAD VIRTUAL "CASI TOTAL"?}

Pero resulta muy forzado pensar que el Universo al completo sea una realidad virtual. Para debilitar esta idea vamos a dar un paso atrás. Vamos a pensar que el Universo sea tan sólo una realidad virtual "casi total".

¿Cómo entender esta noción que acabamos de introducir de realidad virtual "casi total"? Podemos definirla del siguiente modo:

Una realidad virtual casi total es una realidad virtual producida, o generada, o constituida, por un mínimo de realidad no-virtual accesible, por un mínimo de realidad "real" desde nuestros puntos de vista podemos decir también.

Las anteriores posibilidades P-1 (El universo puede ser algo creado por la realidad cuántica) y P-2 (El universo puede ser la ejecución de un gran programa informático) mantienen su vigencia. Pero esta vez, lo que produce, o genera, o constituye, la realidad virtual "casi total" del Universo sí es accesible a algunos de

${ }^{23}$ Pueden encontrarse abundantes análisis sobre los puntos de vista y las perspectivas en Liz (ed.) (2013) y Vázquez y Liz (eds.) (2015). 
nuestros puntos de vista. Habría un mínimo de realidad real que nos es accesible. Y ahora, la pregunta es:

¿Qué puede ser accesible desde nuestros puntos de vista y producir una realidad virtual casi total?

Veamos cómo responder a esta pregunta desde P-1 y P-2. Lo que tendríamos en P-1 sería cierta realidad cuántica con la disposición particularmente vinculada a proyectar el resto del Universo como una realidad virtual. Ciertamente, esa disposición particularmente vinculada puede considerarse realidad virtual en uno de los sentidos examinados. Puede considerarse realidad virtual en el sentido de ser una potencialidad. Pero también debe haber algo más. Al menos, debe haber otras disposiciones no vinculadas exclusivamente a la proyección del resto del universo. Accederíamos a ellas desde ciertos puntos de vista, en particular los que ofrece nuestra física teórica. Esto constituiría la realidad real mínima. Todo lo demás sería realidad virtual. Y el Universo en su conjunto sería una realidad virtual "casi total".

A su vez, lo que tendríamos en P-2 sería cierta realidad computacional con la disposición particularmente vinculada a producir, o generar, o constituir, el resto del Universo como una realidad virtual al ejecutarse cierto programa. Pero esa realidad computacional debería también incluir algo más, otras disposiciones no vinculadas exclusivamente a la ejecución del programa. Esto constituiría ahora la realidad real mínima. Accederíamos a ella desde ciertos puntos de vista. Todo lo demás sería realidad virtual. Y el Universo en su conjunto sería así una realidad virtual "casi total".

¿A qué nos comprometen esos mínimos de realidad real? ¿Cómo de pequeño podría ser ese mínimo? La respuesta es que ese mínimo se obtiene con la existencia de ciertas disposiciones no-vinculadas. Un mínimo de realidad no-virtual, de realidad real, debería ser "un mínimo de disposiciones no-vinculadas a esa realidad virtual".

Y, ¿cómo puede existir ese mínimo de disposiciones no-vinculadas si todo lo demás, absolutamente todo lo demás, es realidad virtual? Una respuesta sería la siguiente. Necesitamos que esas disposiciones no vinculadas sean "puras disposiciones", en el sentido de ser disposiciones que no dependan de nada que no sea una disposición. ${ }^{24}$

24 Podemos distinguir entre "puras" disposiciones (disposiciones que no dependen de nada que no sea una disposición) y disposiciones "absolutas" (disposiciones que no dependen de nada). En la literatura sobre estos temas suele utilizarse la segunda noción. Pero en el contexto del presente trabajo, nos bastaría con la primera. No necesitamos comprometernos con la 
Cualquier otra cosa sería una realidad real "que ya no es mínima". Lo mínimo son unas disposiciones puras no-vinculadas. ${ }^{25}$ Esas disposiciones puras no-vinculadas se manifestarían produciendo o generando la realidad virtual del resto del universo, siendo las disposiciones vinculadas a esta realidad virtual una parte o aspecto de esas disposiciones puras no-vinculadas.

Puede que todo esto de las disposiciones resulte muy complejo. Y también aburrido. Sin embargo, la concepción que se insinúa es sumamente interesante. En el caso del Universo como una realidad virtual "casi total", las relaciones entre una realidad real mínima, constituida por disposiciones no-vinculadas exclusivamente a ese despliegue virtual, y todo lo demás, la gran realidad virtual, serían como las relaciones entre una pura disposición y su manifestación. La realidad real mínima sería una pura disposición no vinculada a la realidad virtual del resto del Universo, acaso un entramado de puras disposiciones. Ciertamente, tal realidad virtual sería la manifestación de ciertas disposiciones particularmente vinculadas, pero también sería, y esto es lo importante, manifestación de esas otras disposiciones puras no vinculadas.

\section{Otras POSIBILIDADES PARA EL UNIVERSO Y PARA LA MENTE}

Si nos tomamos en serio la posibilidad de que casi todo el Universo sea una realidad virtual, y damos a ese "casi todo" la máxima amplitud posible (alternativamente, si reducimos al mínimo, como lo hemos estado haciendo, lo que puede "quedar fuera") la realidad real mínima no podrá ser otra cosa más que una pura disposición no vinculada a esa virtualidad general, o tal vez un entramado de tales puras disposiciones.

A medida que disminuyamos la amplitud del "casi todo" (alternativamente, a medida que ampliemos lo que puede "quedar fuera"), tendremos muchas más

\footnotetext{
existencia de disposiciones absolutas. Acaso no haya nunca necesidad de comprometernos con ellas. Tal vez no podamos llegar nunca al fondo de la realidad. Tal vez la realidad misma no tenga fondo. Es más, tal vez incluso ocurra que la realidad misma no tenga fondo porque nosotros no podemos llegar nunca a un fondo (de hecho, esto último resultará muy plausible a quien asuma el dictum perspectivista de Putnam acerca de que la mente y el mundo construyen conjuntamente la mente y el mundo).

${ }^{25}$ Lo mínimo aquí no puede ser simplemente una colección de objetos. Parece tener sentido la idea de disposiciones puras, disposiciones sin objetos, como algo primitivo, pero no la idea de objetos sin ningún tipo de disposiciones. Los objetos sin disposiciones serían completamente inertes.
} 
posibilidades para el Universo. Y también para la mente. El Universo podrá contener una realidad real mucho más variada. Podrá contener la mesa real sobre la que creo estar escribiendo estas líneas, el árbol real que creo ver a través de la ventana, los electrones que realmente atraviesan una particular rendija, que esté realmente lloviendo en París, que mi vecina no pare realmente de estornudar, que se forme realmente cierto agujero negro en algún lugar del universo, etc. El Universo podrá incluir muchas cosas y muchos hechos que no sean ni realidad virtual, con sus disposiciones particularmente vinculadas, ni puras disposiciones no-vinculadas respecto a esa realidad virtual.

Otro tanto cabe decir de la mente. La interpretación puramente disposicional de la mente, incluso una interpretación disposicional no particularmente vinculada, podrá dejar paso a otras interpretaciones que admitan componentes no sólo disposicionales. Podrá dejar paso a otras interpretaciones que incluyan infinidad de objetos y de hechos.

$\mathrm{El}$ abanico de posibilidades es aquí muy grande. En principio, todas nuestras "pretensiones" tienen aquí cabida. Podemos poblar el Universo con todo tipo de entidades, del tamaño de galaxias enteras o de un tamaño subatómico. También, claro, de un tamaño macroscópico ordinario. Y también podemos poblar nuestra mente con todo tipo de entidades, desde conceptos o proposiciones hasta almas sustanciales, pasando por cosas como datos de los sentidos cuya fuente sean objetos de un mundo externo a nuestra mente. Y todas esas entidades podrán llegar a constituir, en un sentido completamente literal, innumerables hechos.

El gran problema con todas estas opciones no-disposicionales es que tal vez ya no podamos dejar de tomar en serio ese escenario en el que casi todo el Universo sea una inmensa realidad virtual, todo menos justamente ciertas disposiciones puras no exclusivamente vinculadas a esa gran virtualidad. La sospecha sobre las disposiciones, sobre las explicaciones disposicionales tan queridas por los medievales, acompañó al nacimiento mismo de la ciencia moderna. Sin embargo, hoy en día, el lenguaje disposicional es asumido por nuestra ciencia y nuestra técnica. Se ha vuelto indispensable. Y se considera abiertamente que tal vez las descripciones puramente disposiciones sean las descripciones "últimas" en muchos campos. ${ }^{26}$ De esto es de lo que estamos hablando.

${ }^{26}$ Sin duda, la literatura es abundante. Véase, por ejemplo, Cartwright (1989), Crane (ed.) (1996), Groff y Greco (eds.) (2013), Martin (2008), Mellor (1974), Molnar (1999) o Mumford (1998). 
$\mathrm{Y}$ a ello se une otro grupo importante de consideraciones relativas a nuestras mentes. No es fácil dejar de asumir esa concepción de nuestro yo personal, el de cada cual, como una "isla mental" en la que, en último término, toda apariencia no es sino un aspecto de otra apariencia, toda disposición está basada en otras disposiciones, toda mediación se conecta con otras mediaciones y toda construcción utiliza materiales de otras construcciones previas. Acaso esta imagen también sea correcta. Sobre todo, si tenemos en cuenta que estas "islas mentales" pueden no ser precisamente islas "mentales".

Por supuesto, debemos aclarar el último punto. Gran parte de la virtualidad que cabe encontrar en una "isla mental" se genera a través de la experiencia y del lenguaje. Y esto sugiere complejos entrelazamientos de nuestras mentes con algo diferente. Podemos seguir suponiendo que se trata de algo puramente disposicional. Habría tal vez algo así como procesos de acoplamiento puramente disposicionales en los que resultan involucradas tanto disposiciones particularmente vinculadas a las manifestaciones de virtualidad en cada caso relevantes como disposiciones no vinculadas a ellas. ${ }^{27}$ Sea como sea, puede que no todo en nuestras "islas mentales" tenga una naturaleza inequívocamente "mental". Y tanto el lenguaje disposicional como el lenguaje procesual permiten asumir esta idea de una manera mucho más natural y libre de problemas que un lenguaje basado en objetos, o cosas, o entidades, sustanciales que tienen y dejan de tener propiedades.

En cierta ocasión, dejando volar nuestra imaginación sobre estos temas, mi hijo Ulises lo expresó muy bien con la siguiente imagen: "La tierra y el agua salen del barro". La imagen es presocrática, pero tiene un gran valor metafísico. Tal vez haya muchas zonas de barro, muchos terrenos pantanosos. Puede que, en el fondo, toda la realidad, o casi toda, no sea sino un gran terreno pantanoso lleno de un barro primordial.

\section{CUATRO ESCENARIOS RESPECTO A LA VIRTUALIDAD DEL UNIVERSO Y DE LA MENTE}

Al final, nos vemos enfrentados con los siguientes cuatro escenarios respecto a la virtualidad del Universo y de la mente:

\footnotetext{
${ }^{27}$ La noción de procesos de acoplamiento entre disposiciones tiene un papel central en los planteamientos sobre la mente de Martin (2008), que a su vez sintonizan perfectamente con la hipótesis, formulada inicialmente por Clark y Chalmers (1998), de una cognición extendida, o incluso de una mente extendida.
} 
1. No podremos nunca "entender" esos casos límite de virtualidad. Ni el Universo ni la mente pueden ser pensados como virtuales. Y en consecuencia, no puede haber ningún problema genuino respecto de estos casos. Sencillamente, no deberían preocuparnos.

2. Es posible que "todo" eso que queremos llamar Universo, absolutamente todo, no sea sino una gran realidad virtual. $\mathrm{Y}$ de aquí podemos inferir que también lo sería la mente, si es que forma parte del Universo. Y que, entonces, no habría ningún punto de vista desde el cual pudiéramos rechazar como falsa la hipótesis de la inteligencia artificial en su sentido fuerte.

3. Es posible que "casi todo" el Universo sea una realidad virtual. El que ese "casi todo" tenga la máxima amplitud nos conduce a asumir un mínimo de disposiciones puras no vinculadas a esa gran virtualidad, disposiciones a las que se añaden las disposiciones puras que sí se vinculan particularmente a ella. Y podemos llegar a asumir un Universo tal como produciendo, o generando, o constituyendo, a través de ciertos "terrenos pantanosos", a través de multitud de acoplamientos disposicionales, lo que hemos denominado "islas mentales".

4. Si restringimos la amplitud del "casi todo", son posibles "otros tipos de combinaciones de realidad virtual y de realidad real, tanto para el Universo como para la mente. En ambos campos podrían proliferar las disposiciones no-vinculadas a la virtualidad relevante. Y además, todo tipo de entidades no-disposicionales (objetos, hechos, etc.) teniendo y dejando de tener todo tipo de propiedades. Incluso podría llega a considerarse que "casi nada" en el Universo y "casi nada" en la mente es propiamente realidad virtual.

El primero de estos escenarios es básicamente epistemológico, los restantes son descaradamente metafísicos. Pero el segundo escenario también tiene consecuencias epistemológicas importantes. Como hemos visto, nos deja con una completa indeterminación respecto a la realidad "real" capaz de generar o producir todo el Universo, incluyendo la mente, como una gran realidad virtual. Tal realidad "real" no puede consistir en nada habitual.

El tercer escenario corrige esto a través de una realidad real constituida por un mínimo de puras disposiciones no-vinculadas a la virtualidad existente, puras disposiciones no-vinculadas a las que se sumarían las disposiciones particularmente vinculadas a esa virtualidad. La imagen que resulta del Universo acaso nos resulte un poco extraña. Sobre todo, si estamos acostumbrados a pensar en la realidad como en una colección de objetos, o cosas, o entidades sustanciales, que dan lugar 
a hechos al tener y dejar de tener propiedades. Pero la concepción final a la que llegábamos, acompañada de la imagen de esos "terrenos pantanosos" donde se entrelaza lo mental y lo no mental, puede llegar a tener una gran fuerza.

Son posibles otras muchas combinaciones de realidad virtual y de realidad real. El cuarto escenarios abre las puertas a todas ellas. Tanto el Universo como la mente pueden llenarse de todo tipo de disposiciones no-vinculadas, así como de entidades no-disposicionales constituyendo hechos. Pero todas estas disposiciones, entidades y hechos acaban teniendo una realidad problemática, ya se sitúen en nuestro mundo ordinario, en los átomos, en las lejanas galaxias, o en el interior de nuestra conciencia. Al final, acaban siendo sólo "pretensiones" sobre las que debemos hacer a cada paso angustiosas distinciones apariencia/realidad.

Puestos a elegir, debemos confesar que el escenario que nos parece más preferible es el tercero. El cuarto escenario esconde demasiadas ambiciones realistas. Los escenarios primero y segundo son demasiado escépticos.

Miremos hacia atrás. Comenzábamos analizado la noción de realidad virtual tomando como referencia cuatro significados básicos: apariencia, potencialidad, mediación y construcción. Y dichos significados nos parecían perfectamente compatibles y mutuamente enriquecedores. A continuación, nuestra atención se fijó en dos posibles casos límite de virtualidad: el Universo y la mente. Hemos discutido en detalle la posibilidad de que todo el universo sea una realidad virtual. Y también, la posibilidad de que lo sea nuestra mente. Hemos llevado al límite nuestros conceptos. Pero nuestra exploración no nos ha conducido a ninguna respuesta concluyente. Con todo, por el camino sí hemos encontrado algunas cosas interesantes.

Por supuesto, siempre podemos huir de todas estas cuestiones. Siempre podemos esconder la cabeza, como si la cosa no fuera con nosotros. Podemos no hacer ningún esfuerzo por pensar en los casos límite de virtualidad. Pero empeñarse en no entender es condenarse a no entender. Y como decía Heráclito, el carácter de una persona es su destino.

Manuel Liz, Gutiérrez. Universidad de La Laguna manuliz@ull.es 


\section{BibliografíA}

Cartwright, N. (1989): Nature's Capacities and Their Measurement. London: Clarendon Press. Chalmers, D. (2009): “The Matrix as Metaphysics” [http:/ / consc.net/papers/matrix.html]. Chalmers, D. (2017): "The Virtual and the Real". Disputatio, IX, 46: 309-352.

Clark, A. y Chalmers, D. (1998): “The Extended Mind”. Analysis 58, 1: 7-19.

Crane, T. (ed.) (1996): Dispositions. A debate (D.M. Armstrong, C.B. Martin, and U.T. Place). London: Routledge.

Groff, R. y Greco, J. (eds.) (2013): Powers and Capacities in Philosophy. The New Aristotelism, London: Routledge.

Hales, S. (2006): Relativism and the foundations of Philosophy. Cambridge: MIT press.

HeIm, M. (1986): Electric Language: A Philosophical Study of Word Processing. New Haven: Yale Univ. Press.

HeIM, M. (1993): The Metaphysics of Virtual Reality. Oxford: Oxford University Press.

Heim, M. (1998): Virtual Realism. Oxford: Oxford Univ. Press.

Martin C. B. (2008): The Mind in Nature. Oxford: Oxford Univ. Press.

Mellor, D. (1974): "In defense of Dispositions". Philosophical Review 83: 157-181.

Molnar, G. (1999): “Are Dispositions Reducible?”. Philosophical Quarterly 49: 1-17.

Mumford, S. (1998): Dispositions. New York: Oxford Univ. Press

LIz, M. (ed.) (2013): Puntos de vista. Una investigación filosófica. Barcelona: Laertes.

LIZ, M. (2020): “¿Un mundo nuevo? Realidad virtual, realidad aumentada, inteligencia artificial, humanidad mejorada, Internet de las cosas". Arbor, vol 196, no 797.

SeArle, J. (1980): "Minds, Brains, and Programs". The Behavioral and Brain Sciences, 3.

SEARLE, J. (1985): Minds, Brains and Science. The 1984 Reith Lectures. Cambridge: Harvard Univ. Press [Mentes, cerebros y ciencia, Madrid, Cátedra, 1985].

SEARLE, J. (1990): “¿Es la mente un programa informático?”. Investigación y Ciencia, marzo.

SosA, E: (2009): Reflective Knowledge. Apt Belief and Reflective Knowledge, vol. II. Oxford: Clarendon Press.

VÁzquez, M. y Liz, M. (eds.) (2015): Temporal Points of View. Subjective and Objective Aspects, Heidelberg: Springer. 